\title{
Primary cutaneous coccidioidomycosis in an immunocompetent oil field worker in West Texas
}

\author{
Eric Christensen, Mark Feldman* \\ Department of Internal Medicine, Texas Health Presbyterian Hospital, Dallas, Texas, United States
}

Received: June 13, 2019

Accepted: August 12, 2019

Online Published: August 16, 2019

DOI: $10.5430 /$ crim.v6n3p21

URL: https://doi.org/10.5430/crim.v6n3p21

\begin{abstract}
Coccidioidomycosis is a disease endemic to the southwest United States, as well as Central and South America, and primarily causes pulmonary infection. The cutaneous findings associated with Coccidioides species often present as an exanthema or as dissemination from a primary pulmonary infection. Rarely do individuals present with primary cutaneous coccidioidomycosis due to direct inoculation of the skin by arthroconidia. We report a case in which an immunocompetent oil field worker presented with a severe, fungating nasal lesion, who with culture, serology, histopathology, and a convincing history of exposure revealed a diagnosis of primary cutaneous coccidioidomycosis.
\end{abstract}

Key Words: Cutaneous coccidioidomycosis, Coccidioides species, Fungal disease

\section{INTRODUCTION}

Coccidioidomycosis, also known as "Valley Fever" from California's San Joaquin Valley, is a fungal disease endemic to the Southwestern United States, parts of Mexico, and Central and South America. ${ }^{[1]}$ There are an estimated 100,000 to 150,000 cases of infection annually in the United States alone . ${ }^{[1]}$ Coccidioides immitis predominates in Southern California and Northern Mexico, while Coccidioides posadasii is responsible for disease in the other endemic regions. ${ }^{[1]}$ While the two differ genetically, they are clinically and microbiologically indistinguishable. ${ }^{[2]}$ The disease was first described in the late 1800's in Argentina by Dr. Alejandro Posadas, but it wasn't until the 1930's when the broad clinical spectrum of coccidioidomycosis was appreciated. ${ }^{[3,4]}$ The Coccidioides species are dimorphic, soil-dwelling fungi capable of causing systemic disease through inhalation of airborne arthroconidia from the soil. Primary pulmonary coccidioidomycosis is the most common site of infection, with an estimated $60 \%$ of cases being asymptomatic. The other roughly $40 \%$ of affected individuals present with pulmonary and often systemic manifestations that usually appear one to three weeks after inhalation of the organism. ${ }^{[2]}$ Cutaneous manifestations of coccidioidomycosis can be seen in three forms, most commonly as an exanthema of acute pulmonary infection, but also as a secondary cutaneous infection from hematogenous dissemination or as a primary cutaneous infection. Primary cutaneous coccidioidomycosis is an extraordinarily rare disease manifestation, and is the result of direct inoculation into the skin of infectious arthroconidia by an external source. ${ }^{[2]}$ We present a case of an immunocompetent adult male oil field worker who presented with a severe primary cutaneous infection with culture and histopathologically diagnosed coccidioidomycosis.

\section{Case presentation}

A 43-year-old previously healthy African American male presented for evaluation of a fungating, purulent nasal lesion (see Figures 1A, B). The patient was an oil field worker in

\footnotetext{
* Correspondence: Mark Feldman; Email: MarkFeldman@texashealth.org; Address: Department of Internal Medicine, Texas Health Presbyterian Hospital, Dallas, Texas, United States.
} 
West Texas. For this job he operated heavy machinery and regularly had sand and particulate matter blown into his face. He wore eye protection, but not a full-face mask. He denied a history of boils or rashes, cough, chronic rhinosinusitis, nasal cocaine use, a family history of autoimmune disease, and recent travel.

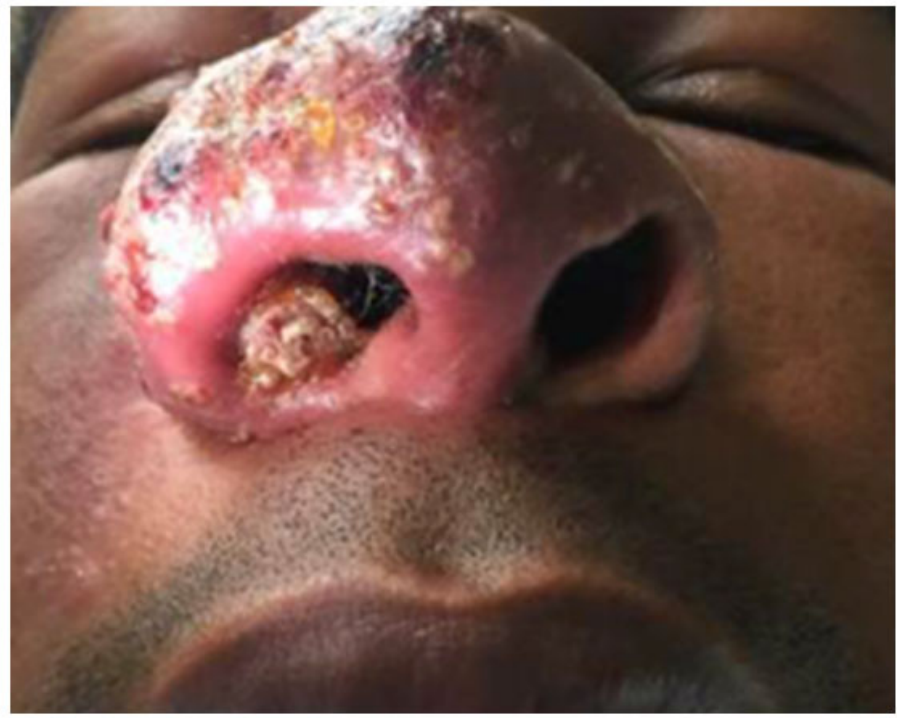

A

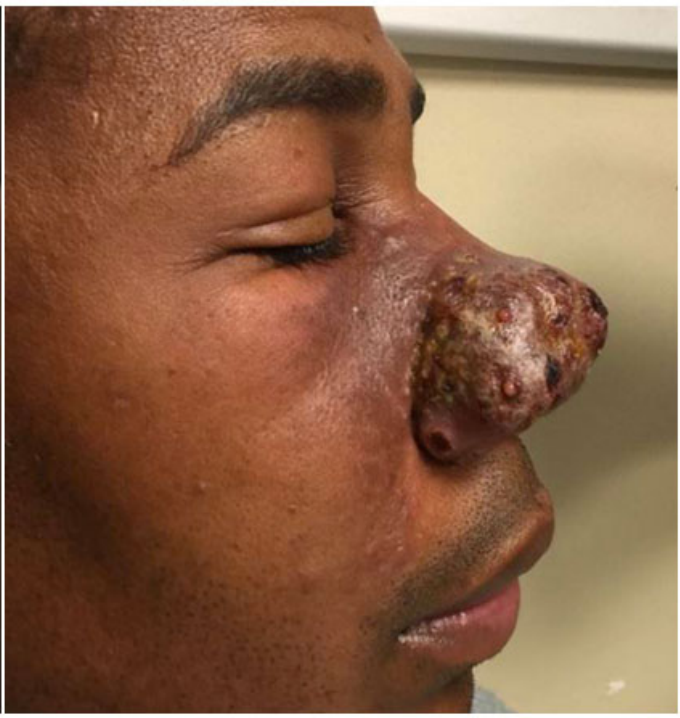

B

Figure 1. 1A. The patient displays a grossly edematous nasal lesion with numerous pustules and superficial crusting. Also note the nodule within the right nostril. 1B. A right, sagittal view captures the diffuse involvement of the nose.

The lesion began 4 weeks earlier with what he believed was a small pimple on the right nasal ala. He popped this lesion and attempted to drain it himself, but then sought medical advice when it enlarged and became more inflamed. He visited multiple urgent care clinics in West Texas and was treated with trimethoprim-sulfamethoxazole, clindamycin, and prednisone. Within a few days of starting the medications, he complained of a diffuse pruritic rash. Clindamycin was replaced by cephalexin, diphenhydramine was added, and he continued on trimethoprim-sulfamethoxazole. After 7 days of outpatient management, he continued to have progressive nasal symptoms in addition to edema under his eyelids, subjective swelling of his anterior cervical lymph nodes, night sweats, and fever. The patient then presented to another facility and, in addition to restarting trimethoprimsulfamethoxazole and cephalexin, he was also given empiric valacyclovir for concern about HSV infection. No definitive diagnostic testing was performed at that time. After a further 7 days of unsuccessful treatment, the patient presented to his PCP, who then directed him to our emergency department, from where he was admitted.

On examination (see Figures 1A, B), the patient was found to have a grossly edematous and erythematous nose affecting the right side more than the left, with multiple pustules and diffuse areas of crusting. There was a nodular mass within the right lateral nostril with serosanguinous drainage present. The patient also had bilateral infra-orbital edema. Anterior cervical lymphadenopathy was present.

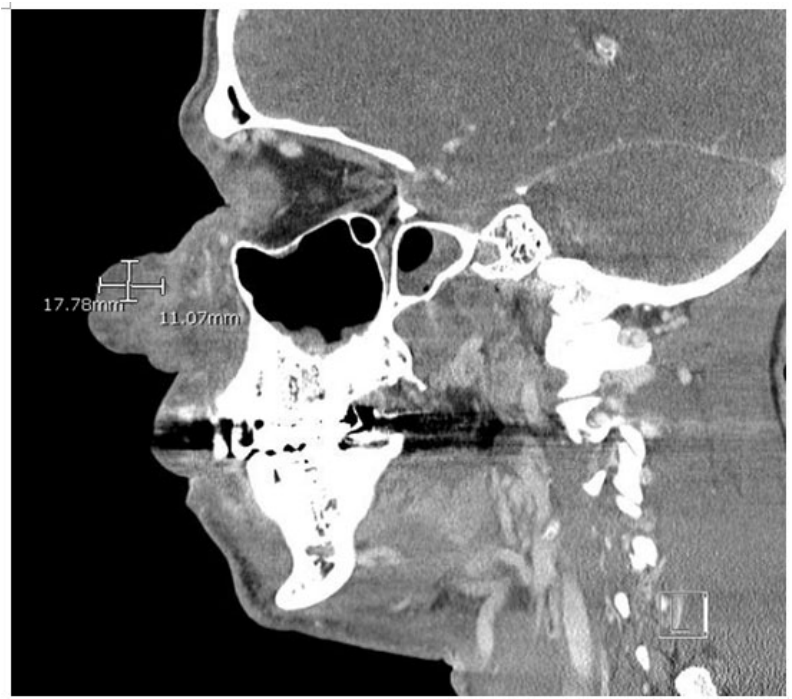

Figure 2. Lateral CT scan of face shows a multi-loculated, poorly defined fluid collection is present with a well-defined peripheral enhancing wall suggesting phlegmon versus early developing abscess involving the right nose, measuring approximately $1.7 \mathrm{~cm} \times 1.5 \mathrm{~cm} \times 1.1 \mathrm{~cm}$. 
For this apparently immunocompetent patient with a fungating purulent dermatitis of the nose, the differential diagnosis included a fungal infection (coccidioidomycosis, blastomycosis, histoplasmosis), a chronic granulomatous bacterial infection (botryomycosis), lymphoma, and sarcoidosis.

Initial laboratory tests included a leukocytosis $(15 \mathrm{k} / \mathrm{ul})$ with eosinophilia $(2.4 \mathrm{k} / \mathrm{ul})$, a peripheral blood smear showing mature eosinophilia, ESR $110 \mathrm{~mm} / \mathrm{hr}(<20 \mathrm{~mm} / \mathrm{hr})$, serum
CRP $7 \mathrm{mg} / \mathrm{dl}(<0.5 \mathrm{mg} / \mathrm{dl})$, and negative HIV p24 antigen and HIV-1,2 antibodies. Chest X-ray was normal. CT of the face with contrast showed prominent facial and nasal cellulitis with an associated multi-loculated poorly defined fluid collection, suggesting a phlegmon or an early-developing abscess of the right nostril (see Figure 2), as well as multiple enlarged, likely reactive submandibular and cervical lymph nodes.

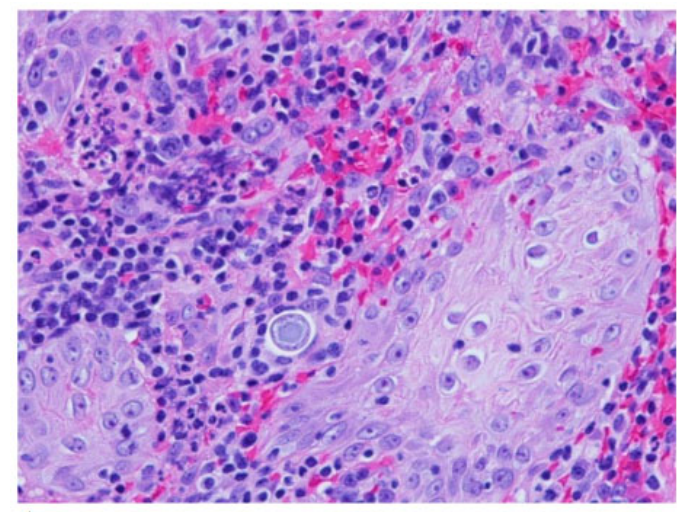

A

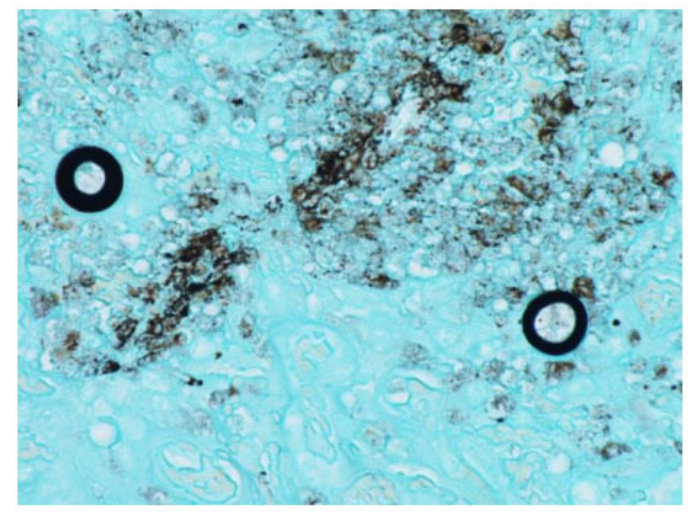

B

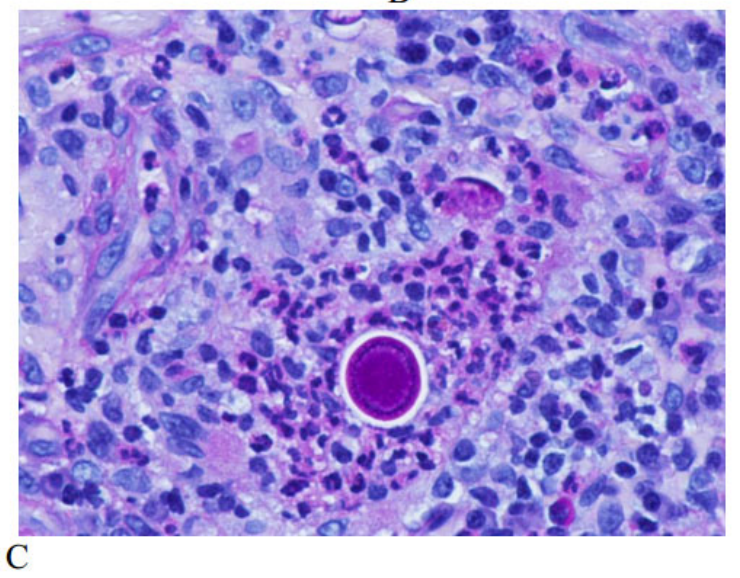

Figure 3. 3A. Section of skin showing inflammation (neutrophils, eosinophils and giant cells) with a refractile Coccidioides spherule. (Hematoxylin \& Eosin. 400x.) 3B. Depicted are thick black-lined Coccidioides spherules. (Gomori Methenamine-Silver Nitrate. 600x.) 3C. Faintly-defined endospores are present within the Coccidioides spherule. (Periodic acid-Schiff. 600x)

The patient was initially started on broad-spectrum antibiotics and the patient was seen by Infectious Disease, Dermatology, and ENT consultants. A bedside Tzanck smear was negative. Nasal swab for MRSA PCR was also negative. The patient was taken to the operating room on day 2 for biopsy of the skin lesion and debridement. Histopathology showed extensive benign epithelial hyperplasia, ulceration, and marked acute inflammation with admixed patchy granulomatous inflammation with small collections of histiocytes and multinucleated histiocytic giant cells and numerous eosinophils. Periodic acid-Schiff, Hematoxylin \& Eosin, and
Gomori methenamine silver stains demonstrated fungal organisms with large endosporulating spherules, compatible with coccidioidomycosis (see Figures 3A, B, C). Fungal cultures revealed a Coccidioides species, and serum complement fixation titers for Coccidioides were positive at a titer of 1:4 (titers of less than 1:32 may indicate past infection or self-limited disease; titers greater than or equal to 1:32 may indicate disseminated infection).

The patient was started on fluconazole $800 \mathrm{mg}$ po daily, and was discharged with follow up by wound care, ENT, and infectious disease. Three weeks after discharge, the patient 
was noted to have decreased swelling of the nose, with no pustular lesions. He was continued on fluconazole $800 \mathrm{mg}$ for 8 weeks, followed by $400 \mathrm{mg}$ for an additional 4 months. Three months after presenting to our hospital, the patient was noted to have complete resolution of his fungating lesion with minimal scarring. He returned to work, and began using a mask covering his nose and mouth, in addition to eye protection, but not a full face shield.

\section{Discussion}

Coccidioidomycosis continues to be a diagnostic challenge to healthcare professionals. As of 2019, it is a reportable disease in 27 U.S. states, with roughly 10,000 U.S. cases reported annually, but with an estimated incidence 6 to 14 times higher, and steadily increasing since it became reportable in the 1990's. ${ }^{[5]}$ Some suspected endemic states, such as Texas, Oklahoma, and Colorado do not report cases. Observed increases in incidence may be attributable to improved reporting, better diagnostic tools, changing climate, increased construction and soil disturbance, and population growth. ${ }^{[3]}$ Those at greater risk for exposure include people involved in construction work, farm work, archeological digs, and also prison inmates and guards. ${ }^{[3]}$ In addition, populations known to have greater risk for severe disease include African Americans, Filipinos, pregnant women, and immunosuppressed individuals, such as those with HIV/AIDS, hematologic malignancies, inflammatory arthritides, diabetes mellitus, as well as those with solid organ transplantation and subsequent use of immunosuppressive agents. ${ }^{[2]}$ Our patient had a workrelated exposure in an underreported, likely endemic area, and was of a racial background (African American) known to carry increased risk for infection and spread. Most cases of symptomatic coccidioidomycosis include individuals who experience mild to moderate influenza-like symptoms, including arthralgias, myalgias, fever, fatigue, and cough. ${ }^{[4]}$ Approximately $40 \%$ of exposed individuals have a presentation consistent with community-acquired pneumonia, in which the chest radiograph shows lobar infiltrates that, in the minority of cases, become chronic progressive pulmonary disease with nodules or cavitations. ${ }^{[6]}$ The skin is the most common extra-pulmonary site of infection and may be the only manifestation of disseminated coccidioidal disease. The cutaneous findings associated with coccidioidomycosis are categorized as reactive eruptions and organism-specific lesions. ${ }^{2]}$ Reactive eruptions do not contain the organism, and occur during acute primary pulmonary infection in up to $50 \%$ of individuals. ${ }^{[2]}$ Reactive eruptions include erythema nodosum, an acute generalized exanthema, erythema multiforme, Sweet's syndrome, and reactive interstitial granulomatous dermatitis. ${ }^{[4]}$ Organism-specific lesions result from hematogenous dissemination to the skin, or more rarely from primary cutaneous infection, and contain the organism which can be identified by histopathologic staining or by culture. The cutaneous findings of organism-specific lesions are diverse and include macules, papules, pustules, nodules, ulcers, verrucous plaques, abscesses, and atrophic scars. ${ }^{[7]}$ Primary cutaneous coccidioidomycosis is extremely rare, and is believed to occur secondary to a traumatic inoculation of the organism from direct contact with a source in the environment or in the laboratory. Cases have been reported among agricultural workers, laboratory workers, and those with splinter injuries or lacerations. ${ }^{[4]}$ If a patient's pulmonary symptoms are minimal or absent, it may be difficult to distinguish primary cutaneous infection from disseminated infection, but supporting clues may include regional lymphadenopathy, a relatively low complement fixation titer and a history of trauma-induced skin injury. ${ }^{[4]}$ Diagnostic criteria for primary cutaneous infection were established in 1953 by Wilson et al. and include the following: absence of pulmonary disease, evidence of traumatic inoculation, a 1 to 3 week incubation period, a chancriform lesion with a painless, ulcerated nodule or plaque; an early positive anti-IDTP (Immunodiffusion Tube Precipitin) IgM , an early positive coccidioidin skin test, a negative or low complement fixation test, the presence of regional lymphadenopathy and sporotrichoid nodules, and spontaneous healing within a few weeks. ${ }^{[2]}$ In the case presented, the patient did not cite a history indicative of pulmonary involvement, and his chest x-ray was normal. Although there was no specific and solitary event of inoculation, the patient stated he regularly had soil and dust particles blasted into his face at high velocities, which would support either primary cutaneous or primary lung infection with cutaneous dissemination. Coccidioides antibody by complement fixation was positive, yet in low titer, which is fitting with primary cutaneous infection. And finally, the morphology of his facial lesion, including painful suppuration and regional lymphadenopathy may favor primary cutaneous infection, but is not exclusive to it. ${ }^{[8]}$

Diagnosis of cutaneous lesions is made chiefly from histopathological examination of biopsied lesions, with usual findings including granulomatous inflammation with large, thick-walled spherules that may or may not contain endospores. ${ }^{[7]}$ The inflammatory reaction to endospores is mostly neutrophilic, while the reaction to spherules is granulomatous. ${ }^{[6]}$ Eosinophils may also be present, and can produce the Splendore-Hoeppli phenomenon, which is an intense rim of eosinophilic material around the fungal elements. ${ }^{[6]}$ The sensitivity for histopathologic detection of Coccidioides species is roughly $84 \% .{ }^{[6]}$ Biopsied material should also be sent to the microbiology lab for culture as the 
organism can readily grow on most mycologic or bacteriologic media, which occurred in our case as well. ${ }^{[4]}$ Serologic assays for antibodies to Coccidioides antigens can also be used, but can be insensitive in immunocompromised patients. ${ }^{[7]}$ Finally, antigen assays for galactomannan can be helpful in the acute setting, although there is cross-reactivity with Histoplasma species. ${ }^{[7]}$

The prognosis of primary cutaneous infection is usually favorable, with most cases self-resolving. ${ }^{[8]}$ The range of involvement can be highly variable, as in the case presented, in which surgical debridement of abscesses is indicated. ${ }^{[2]}$
Treatment is otherwise medical, and includes 3 to 6 months of daily fluconazole or itraconazole.

Although coccidioidomycosis is a well-known disease to most clinicians, its variable presentation, specifically with only cutaneous involvement, can make diagnosis difficult. A focused and thorough history, in addition to timely and appropriate diagnostic testing can improve outcomes in these patients.

\section{CONFLicts OF INTEREST Disclosure}

The author declares no conflict of interest

\section{REFERENCES}

[1] Shiu J, Thai M, Elsensohn A, et al. A case series of primary cutaneous coccidioidomycosis after a record-breaking rainy season. JAAD Case Reports. 2018; 4(5): 412-414. PMid:29984266. https://doi.org/10.1016/j.jdcr.2017.11.018

[2] Garcia S, Flores M, Cabrera L, et al. Coccidioidomycosis and the skin: a comprehensive review. Anais Brasileiros de Dermatologia. 2015; 90(5): 610-619. PMid:26560205. https://doi .org/10.1 $590 /$ abd1806-4841.20153805

[3] Kollath D, Miller K, Barker B. The mysterious desert dwellers: Coccidioides immitis and Coccidioides posadasii, causative fungal agents of coccidioidomycosis. Virulence. 2019; 10(1): 222-233. PMid:30898028. https://doi.org/10.1080/21505594. 2019. 1589363

[4] DiCaudo D. Coccidioidomycosis: a review and update. Journal of the American Academy of Dermatology. 2006; 55(6): 929-942. PMid:17110216. https://doi.org/10.1016/j.jaad.2006.04 .039

[5] McCotter O, Benedict K, Engelthaler D, et al. Update on the Epidemiology of coccidioidomycosis in the United States. Medical Mycology. 2019; 57: S30-S40. PMid:30690599. https://doi .org/10.109 $3 / \mathrm{mmy} / \mathrm{myy} 095$

[6] Guarner J, Brandt M. Histopathologic diagnosis of fungal infections in the 21st century. Clinical Microbiology Reviews. 2011; 24(2): 247-280. PMid:21482725. https://doi.org/10.1128/CMR.00 053-10

[7] Smith J, Riddell J, Kauffman C. Cutaneous manifestations of endemic mycoses. Current Infectious Disease Reports. 2013; 15(5): 440-449. PMid:23917880. https://doi.org/10.1007/s11908 $-013-0352-2$

[8] Russell D, Ager E, Wohltman W. Cutaneous coccidioidomycosis masquerading as an epidermoid cyst: case report and review of the literature. Military Medicine. 2017; 182(1/2): e1665-e1668. PMid:28051991. https://doi .org/10.7205/MILMED-D-15-0 0549 